\title{
A MODEL OF ULTRASONIC TESTING FOR CRACKS NEAR A NON-PLANAR SURFACE
}

\author{
Jonathan Westlund and Anders Boström \\ Department of Applied Mechanics, Chalmers University of Technology, \\ SE-412 96 Göteborg, Sweden
}

\begin{abstract}
Based on the methodology of the 2D models presented at QNDE 2008 and QNDE 2009, this paper presents an extension to 3D of a model of ultrasonic testing for cracks near a non-planar back surface. The elastic wave scattering in a thick-walled component by an interior rectangular crack located near a back surface of arbitrary geometry is considered. The 3D wave scattering problem is solved using a hybrid boundary integral equation method (BIEM): a displacement boundary element method (BEM) for the back surface displacements is combined with an analytical technique for the hypersingular traction integral equation for the crack-opening displacements. The action of ultrasonic transducers in transmission and reception is taken into account in the model, and a few numerical examples illustrating the influence of the back surface geometry are given.
\end{abstract}

Keywords: Ultrasonics, Modeling, Crack, BIE, BEM

PACS: 43.20.Gp, 43.20.Rz, 43.35.Zc.

\section{INTRODUCTION}

Quantitative methods of nondestructive evaluation (NDE) today play an important role in in-service inspection and maintenance of structures, with especially important applications in the aerospace and nuclear industries where failures can have very severe consequences. Among the several methods of NDE that exist today the ultrasonic techniques, the focus of the present paper, are arguably the most important.

Common to all methods of NDE is the need of a measurement model, i.e. a model of the complete testing situation, and the purpose of the present work is to develop such a model for the case of ultrasonic testing for cracks located close to a non-planar back surface. This situation occurs e.g. in the nuclear power industry in the testing of thick-walled pipes featuring diameter changes or connections. The interaction between the non-planar back surface and the defect then complicates the testing because the signal response from the defect may be masked by the response from the back surface.

The solution method employed in the model is based on boundary integral equation methods, BIEMs, with the major advantage of being essentially exact methods such that the results are valid both for low, intermediate and high frequencies. This is in contrast to approximate theories such as the Geometrical Theory of Diffraction (GTD) and the elastodynamic

Review of Progress in Quantitative Nondestructive Evaluation, Volume 30
AIP Conf. Proc. 1335, 75-82 (2011); doi: 10.1063/1.3591842

(C) 2011 American Institute of Physics 978-0-7354-0888-3/\$30.00 
Kirchhoff theory. These approximate theories provide powerful and efficient methods with the possibility to treat complex geometries, but finding the bounds of applicability of the theories in a specific case can be difficult. The identification of such bounds may actually be one of the important areas of application of an essentially exact model, as discussed in the paper by Schafbuch et al [1].

\section{PROBLEM FORMULATION}

The scattering geometry is depicted in Fig. 1, where an interior rectangular crack with sides $2 a_{1}$ and $2 a_{2}$ is located in a thick-walled component with a non-planar back surface. Conventional transmitting and receiving ultrasonic contact probes $(\mathrm{T})$ and $(\mathrm{R})$, respectively, scan along the surface of the component. The same probe may also be used in both transmission and reception, in the common case of pulse-echo testing.

The figure also introduces four coordinate systems: the crack coordinate system $\boldsymbol{x}^{\mathrm{c}}$, the back surface system $\boldsymbol{x}^{\mathrm{b}}$, the transmitter system $\boldsymbol{x}^{\mathrm{t}}$ and the receiver system $\boldsymbol{x}^{\mathrm{r}}$. The superscripts ' $c$ ', ' $b$ ', ' $t$ ' and ' $r$ ' on quantities indicate that they are represented in the corresponding coordinate system. Omitted superscripts indicate expressions which hold in any of the coordinate systems.

The location of the crack centre relative the back surface coordinate system is given by the vector $\boldsymbol{d}$, and the crack orientation relative the back surface coordinate system is described by the three Euler angles $\left(\varphi^{\mathrm{c}}, \theta^{\mathrm{c}}, \psi^{\mathrm{c}}\right)$. Here $\varphi^{\mathrm{c}}$ is the rotation around the $x_{3}^{\mathrm{b}}$-axis, $\theta^{\mathrm{c}}$ the rotation around the intermediate $x_{2}$-axis and $\psi^{\mathrm{c}}$ the rotation around the $x_{3}^{\mathrm{c}}$-axis. The standard transformation rules for the transformation between the coordinate systems apply, with the rotation matrix $\mathrm{R}^{\mathrm{c}}$ from $\boldsymbol{x}^{\mathrm{b}}$ to $\boldsymbol{x}^{\mathrm{c}}$. The positions of the transmitting and receiving probes relative the back surface coordinate system are given by the vectors $\boldsymbol{d}^{\mathrm{T}}$ and $\boldsymbol{d}^{\mathrm{R}}$, respectively, and the probe systems and the back surface system are assumed to be collinear.

The shape of the back surface is described by the function $g\left(x_{1}^{\mathrm{b}}, x_{2}^{\mathrm{b}}\right)$ which is allowed to be quite arbitrary, as long as it features no cusps which would introduce additional back surface scattering not accounted for in the model. In the subsequent numerical solution procedure the infinite back surface is truncated and discretized, and $\left(T_{1}, T_{2}\right)$ and $\left(T_{3}, T_{4}\right)$ denote the corresponding lower and upper truncation limits in the $x_{1}^{\mathrm{b}}$ - and $x_{2}^{\mathrm{b}}$-directions, respectively.

The component is linearly elastic, isotropic and homogeneous in the exterior of the crack, with Lamé constants $\lambda$ and $\mu$ and density $\rho$. The distance between the crack and the back surface may be arbitrary as long as the crack is interior, but the distance between the scanning surface of the component and the crack and back surface is assumed to be at least a couple of wavelengths so that multiple scattering between these surfaces may be neglected.

Only time-harmonic elastodynamics is considered and the time-factor $\mathrm{e}^{-\mathrm{i} \omega t}$, with $\omega$ denoting the angular frequency and $t$ the time, is suppressed throughout. The elastodynamic equations of motion are then:

$$
k_{p}^{-2} \nabla(\nabla \cdot \boldsymbol{u})-k_{s}^{-2} \nabla \times(\nabla \times \boldsymbol{u})+\boldsymbol{u}=\mathbf{0},
$$

where $k_{p}=\omega / c_{p}$ is the pressure wave number, $c_{p}=\sqrt{(\lambda+2 \mu) / \rho}$ the pressure wave speed, $k_{s}=\omega / c_{s}$ the shear wave number and $c_{s}=\sqrt{\mu / \rho}$ the shear wave speed. Adding the traction-free boundary conditions on the crack and back surface, a specified incident wave field and the usual outgoing radiation condition at infinity completes the formulation of the wave scattering problem at hand. 


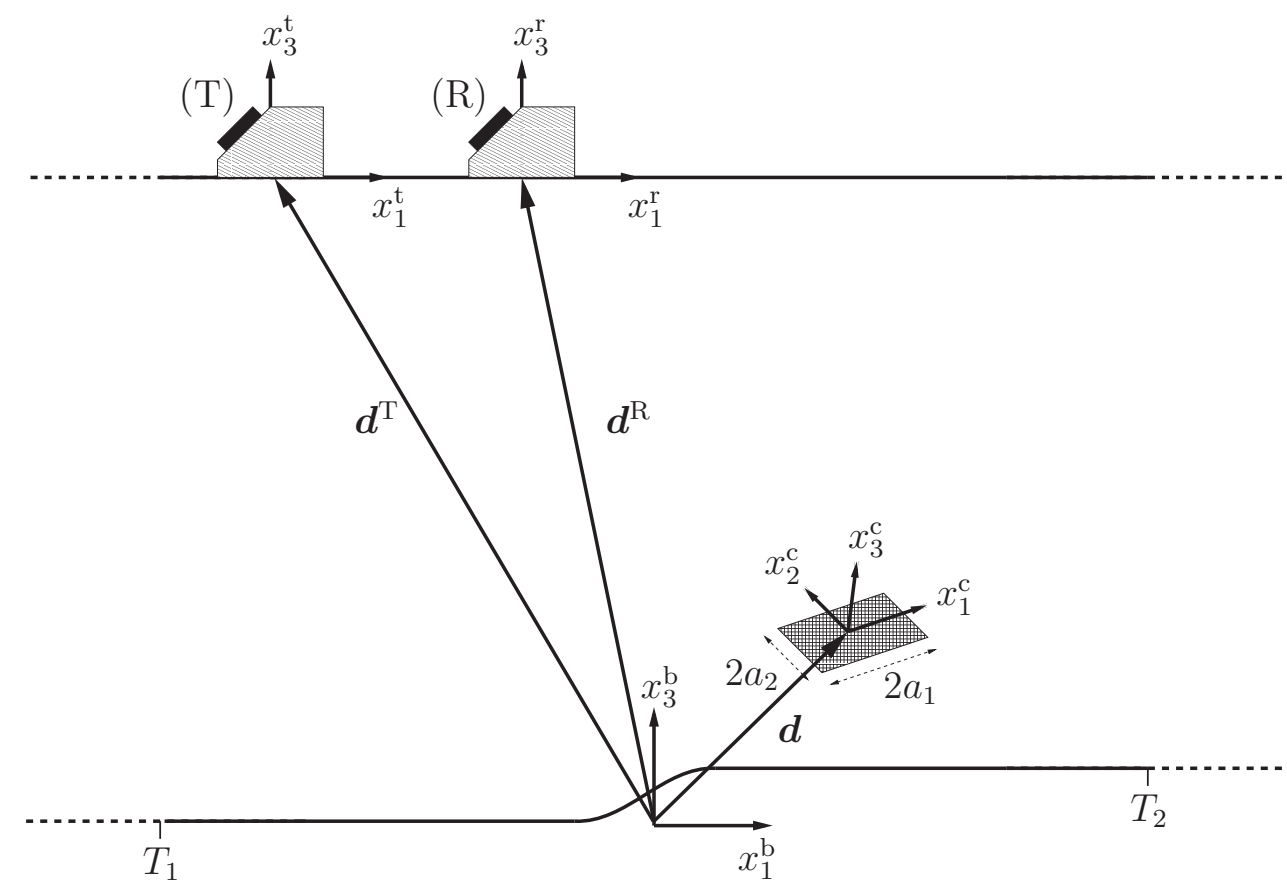

FIGURE 1. The geometry with a rectangular crack in a component with a non-planar back surface.

\section{THE INTEGRAL EQUATIONS}

The wave scattering problem is solved using boundary integral equation (BIE) methods; the scattering problem is reformulated as two coupled BIEs which are then solved simultaneously. The BIE-reformulation is based on use of the isotropic free-space outgoing wave Green's tensor denoted $\boldsymbol{U}^{k}(\boldsymbol{x}, \boldsymbol{y} ; \omega)$ with corresponding stress tensor $\boldsymbol{\Sigma}^{k}(\boldsymbol{x}, \boldsymbol{y} ; \omega) \equiv$ $\mathcal{C}: \nabla \boldsymbol{U}^{k}(\boldsymbol{x}, \boldsymbol{y} ; \omega)$, where $\mathcal{C}$ is the elastic stiffness tensor and $k=1,2,3$ denotes the direction of the applied point load. Throughout the paper, the $\nabla$-operator always acts on the $\boldsymbol{x}$-coordinates. The Green's tensor is given in closed form by e.g. Bonnet [2] and on Fourier integral form by Boström and Bövik [3].

The back surface integral equation may be derived directly using the Green's tensor, the divergence theorem and a limiting process. However, in order to avoid strongly singular integrals an indirect regularization approach (see Bonnet [2]) is also followed. This approach transfers the singularity of the dynamic Green's stress tensor $\boldsymbol{\Sigma}^{k}(\boldsymbol{x}, \boldsymbol{y} ; \omega)$ to the static Green's stress tensor $\boldsymbol{\Sigma}^{k}(\boldsymbol{x}, \boldsymbol{y})=\mathcal{C}: \nabla \boldsymbol{U}^{k}(\boldsymbol{x}, \boldsymbol{y})$, with $\boldsymbol{U}^{k}(\boldsymbol{x}, \boldsymbol{y})$ denoting the corresponding static displacement tensor. This Green's tensor is the corresponding fundamental solution for $\omega=$ 0 , and it is given explicitly by e.g. Bonnet [2]. By transferring the singularity to the static Green's tensor it becomes possible to evaluate the strongly singular integral analytically. The result is a back surface BIE containing no worse than weakly singular integrals, granted that the displacement $\boldsymbol{u}$ satisfies the usual Hölder-continutity assumption [2].

The derivation of the back surface BIE and the regularization is a straightforward extension to 3D of the results in the paper by Westlund and Boström [4], resulting in the 
following regularized back surface integral equation:

$$
\begin{aligned}
& \int_{S_{T}} u_{i}^{\mathrm{b}}\left(\boldsymbol{x}^{\mathrm{b}}\right)\left[\sum_{i j}^{k}\left(\boldsymbol{x}^{\mathrm{b}}, \boldsymbol{y}^{\mathrm{b}} ; \omega\right)-\sum_{i j}^{k}\left(\boldsymbol{x}^{\mathrm{b}}, \boldsymbol{y}^{\mathrm{b}}\right)\right] \mathrm{n}_{j}^{\mathrm{b}}\left(\boldsymbol{x}^{\mathrm{b}}\right) \mathrm{d} S_{\boldsymbol{x}^{\mathrm{b}}} \\
+ & \int_{S_{T}}\left[u_{i}^{\mathrm{b}}\left(\boldsymbol{x}^{\mathrm{b}}\right)-u_{i}^{\mathrm{b}}\left(\boldsymbol{y}^{\mathrm{b}}\right)\right] \Sigma_{i j}^{k}\left(\boldsymbol{x}^{\mathrm{b}}, \boldsymbol{y}^{\mathrm{b}}\right) \mathrm{n}_{j}^{\mathrm{b}}\left(\boldsymbol{x}^{\mathrm{b}}\right) \mathrm{d} S_{\boldsymbol{x}^{\mathrm{b}}} \\
+ & \mathrm{R}_{i p}^{\mathrm{c}} \mathrm{R}_{3 q}^{\mathrm{c}} \int_{-a_{2}}^{a_{2}} \int_{-a_{1}}^{a_{1}} \Delta u_{i}^{\mathrm{c}}\left(x_{1}^{\mathrm{c}}, x_{2}^{\mathrm{c}}\right) \Sigma_{p q}^{k}\left(\left(\mathrm{R}^{\mathrm{c}}\right)^{\mathrm{T}}\left\{x_{1}^{\mathrm{c}}, x_{2}^{\mathrm{c}}, 0\right\}+\boldsymbol{d}^{\mathrm{b}}, \boldsymbol{y}^{\mathrm{b}} ; \omega\right) \mathrm{d} x_{1}^{\mathrm{c}} \mathrm{d} x_{2}^{\mathrm{c}} \\
- & \frac{1}{2} u_{k}^{\mathrm{b}}\left(\boldsymbol{y}^{\mathrm{b}}\right)+u_{k}^{\mathrm{in}, \mathrm{b}}\left(\boldsymbol{y}^{\mathrm{b}}\right)=0,
\end{aligned}
$$

where $\boldsymbol{y} \in S_{T}, \boldsymbol{u}$ is the total displacement field, $\mathbf{n}$ the upward unit normal vector of the back surface, $\Delta \boldsymbol{u}$ the crack opening displacement (COD): $\Delta \boldsymbol{u}\left(x_{1}^{\mathrm{c}}, x_{2}^{\mathrm{c}}\right) \equiv \boldsymbol{u}\left(\left\{x_{1}^{\mathrm{c}}, x_{2}^{\mathrm{c}}, 0^{+}\right\}\right)-$ $\boldsymbol{u}\left(\left\{x_{1}^{\mathrm{c}}, x_{2}^{\mathrm{c}}, 0^{-}\right\}\right), \boldsymbol{u}^{\mathrm{in}}$ the incident displacement field from the transmitting probe and $k=$ $1,2,3$. In this integral equation the integrals over the infinite back surface have been replaced by integrals over the truncated back surface $S_{T}$. This approximation is expected to be valid for sufficiently large truncation limits $\left(T_{1}, T_{2}\right)$ and $\left(T_{3}, T_{4}\right)$.

The crack integral equation follows immediately from an integral representation of $\boldsymbol{u}$ by applying Hooke's law and invoking the traction-free boundary condition on the crack. The result is the integral equation:

$$
\begin{aligned}
\lim _{y_{3}^{\mathrm{c}} \rightarrow 0} \sigma_{i 3}^{\mathrm{c}}\left(y_{1}^{\mathrm{c}}, y_{2}^{\mathrm{c}}, y_{3}^{\mathrm{c}}\right)=\int_{S_{T}} u_{m}^{\mathrm{c}}\left(\boldsymbol{x}^{\mathrm{c}}\right) \mathcal{C}_{i 3 k l} \frac{\partial}{\partial y_{l}^{\mathrm{c}}} \sum_{m n}^{k}\left(\boldsymbol{x}^{\mathrm{c}},\left\{y_{1}^{\mathrm{c}}, y_{2}^{\mathrm{c}}, 0\right\} ; \omega\right) \mathrm{n}_{n}^{\mathrm{c}}\left(\boldsymbol{x}^{\mathrm{c}}\right) \mathrm{d} S_{\boldsymbol{x}^{\mathrm{c}}} \\
\quad+\lim _{y_{3}^{\mathrm{c}} \rightarrow 0} \int_{-a_{2}}^{a_{2}} \int_{-a_{1}}^{a_{1}} \Delta u_{m}^{\mathrm{c}}\left(x_{1}^{\mathrm{c}}, x_{2}^{\mathrm{c}}\right) \mathcal{C}_{i 3 k l} \frac{\partial}{\partial y_{l}^{\mathrm{c}}} \Sigma_{m 3}^{k}\left(\left\{x_{1}^{\mathrm{c}}, x_{2}^{\mathrm{c}}, 0\right\}, \boldsymbol{y}^{\mathrm{c}} ; \omega\right) \mathrm{d} x_{1}^{\mathrm{c}} \mathrm{d} x_{2}^{\mathrm{c}} \\
\quad+\mathcal{C}_{i 3 k l} \frac{\partial}{\partial y_{l}^{\mathrm{c}}} u_{k}^{\mathrm{in}, \mathrm{c}}\left(\left\{y_{1}^{\mathrm{c}}, y_{2}^{\mathrm{c}}, 0\right\}\right)=0,
\end{aligned}
$$

where $i=1,2,3,\left|y_{1}^{\mathrm{c}}\right|<a_{1},\left|y_{2}^{\mathrm{c}}\right|<a_{2}$ and $\mathcal{C}_{i j k l}$ are the components of the isotropic elastic stiffness tensor $\mathcal{C}$. The second integral in this equation is of the hypersingular type, so the limit cannot be moved inside the integral. However, the present solution method enables an explicit evaluation of the limit at a later stage, as described below.

\section{PROBE MODELING}

In order to account for the action of a conventional ultrasonic contact probe in transmission the probe model developed by Boström and Wirdelius [5] is used. This model is based on prescribing the traction on the scanning surface of the component, and the traction is taken as that due to a plane SH-, SV- or P-wave with given amplitude and propagation direction. For the transmitting probe $(\mathrm{T})$, the boundary condition on the upper surface of the component is then taken as the traction:

$$
\boldsymbol{t}^{\mathrm{t}}= \begin{cases}\mathrm{i} A_{0} f \mu k_{p}\left[\delta \sin 2 \gamma_{\mathrm{t}} \mathbf{e}_{x_{1}^{\mathrm{t}}}+\left(\frac{k_{s}^{2}}{k_{p}^{2}}-2 \sin ^{2} \gamma_{\mathrm{t}}\right) \mathbf{e}_{x_{3}^{\mathrm{t}}}\right] \mathrm{e}^{-\mathrm{i} k_{p} x_{1}^{\mathrm{t}} \sin \gamma_{\mathrm{t}}}, & \text { P probe } \\ \mathrm{i} A_{0} f \mu k_{s}\left[-\delta \cos 2 \gamma_{\mathrm{t}} \mathbf{e}_{x_{1}^{\mathrm{t}}}+\sin 2 \gamma_{\mathrm{t}} \mathbf{e}_{x_{3}^{\mathrm{t}}}\right] \mathrm{e}^{-\mathrm{i} k_{s} x_{1}^{\mathrm{t}} \sin \gamma_{\mathrm{t}}}, & \text { SV probe }, \\ \mathrm{i} A_{0} f \mu k_{s} \delta \cos \gamma_{\mathrm{t}} \mathbf{e}_{x_{2}^{\mathrm{t}}} \mathrm{e}^{-\mathrm{i} k_{s} x_{1}^{\mathrm{t}} \sin \gamma_{\mathrm{t}}}, & \text { SH probe },\end{cases}
$$

beneath the surface of the probe and $\boldsymbol{t}^{\mathrm{t}}=\mathbf{0}$ elsewhere. The surface of the probe is assumed to be either elliptical or rectangular, and the function $f$ is introduced in order to allow for 
a non-constant traction tapering off towards the edges of the probe. The transmitting probe angle $\gamma_{\mathrm{t}}$ is the rotation in the $x_{2}^{\mathrm{t}}$-direction measured from the negative $x_{3}^{\mathrm{t}}$-axis, the parameter $\delta$ accounts for the effect of a couplant applied between the probe and scanning surface and $A_{0}$ is the amplitude of the plane wave.

When multiple reflections between the scanning surface and the crack and back surface are neglected, a prescription of the traction in this fashion enables an explicit solution of the boundary value problem for the incident field using a double Fourier transform. The explicit expressions and all details are given in the paper by Boström and Wirdelius [5] and are not repeated here.

In order to predict the signal response measured in an ultrasonic testing situation, the action of the receiving probe is modelled using the electromechanical reciprocity relation by Auld [6]. Applied to this case the reciprocity relation gives the crack signal response as:

$$
\delta \Gamma_{C}=\frac{-\mathrm{i} \omega}{4 P} \int_{-a_{2}}^{a_{2}} \int_{-a_{1}}^{a_{1}} \Delta u_{i}^{\mathrm{c}}\left(x_{1}^{\mathrm{c}}, x_{2}^{\mathrm{c}}\right) \sigma_{i 3}^{\mathrm{re}, \mathrm{c}}\left(x_{1}^{\mathrm{c}}, x_{2}^{\mathrm{c}}, 0\right) \mathrm{d} x_{1}^{\mathrm{c}} \mathrm{d} x_{2}^{\mathrm{c}},
$$

and the back surface signal response as:

$$
\delta \Gamma_{B S}=\frac{-\mathrm{i} \omega}{4 P} \int_{S_{T}} u_{i}^{\mathrm{re}, \mathrm{b}}\left(\boldsymbol{x}^{\mathrm{b}}\right) \sigma_{i j}^{\mathrm{in}, \mathrm{b}}\left(\boldsymbol{x}^{\mathrm{b}}\right) \mathrm{n}_{j}^{\mathrm{b}}\left(\boldsymbol{x}^{\mathrm{b}}\right) \mathrm{d} S_{\boldsymbol{x}^{\mathrm{b}}}
$$

In these equations the superscript 're' denotes quantities computed with the back surface present but the crack absent and the incoming field generated by the receiving probe acting as transmitter, and the superscript 'in' denotes quantities computed with both back surface and crack absent and the incoming field generated by the transmitting probe. The COD $\Delta u_{i}$ is due to an incoming field from the transmitting probe with both crack and back surface present. The probes are transmitting at the fixed angular frequency $\omega$, and the quantity $P$ is essentially the power supplied to the transmitting probe.

\section{NUMERICAL SOLUTION AND EXAMPLES}

The two coupled boundary integral equations (2) and (3) are solved by discretizing them and subsequently solving the resulting system of linear algebraic equations. For the COD a series expansion in Chebyshev functions is used. The Chebyshev functions are defined by:

$$
\psi_{n}(v)= \begin{cases}\frac{1}{\pi} \cos (n \arcsin v), & n=1,3, \ldots, \\ \frac{1}{\pi} \sin (n \arcsin v), & n=2,4, \ldots,\end{cases}
$$

such that the expansion reads:

$$
\Delta u_{i}^{\mathrm{c}}\left(x_{1}^{\mathrm{c}}, x_{2}^{\mathrm{c}}\right)=\sum_{n_{1}=1}^{N_{1}} \sum_{n_{2}=1}^{N_{2}} \alpha_{i n_{1} n_{2}} \psi_{n_{1}}\left(x_{1}^{\mathrm{c}} / a_{1}\right) \psi_{n_{2}}\left(x_{2}^{\mathrm{c}} / a_{2}\right) .
$$

This expansion allows for an analytical evaluation of the crack integrals arising in the crack BIE, due to the fact that the Chebyshev functions satisfy the integral relation:

$$
\int_{-1}^{1} \psi_{n}(v) \mathrm{e}^{-\mathrm{i} \gamma v} \mathrm{~d} v=\frac{n}{\gamma} \mathrm{J}_{n}(\gamma)
$$

where $\mathrm{J}_{n}$ is the Bessel function of the first kind and order $n$. The expansion also exhibits the correct behaviour at the crack edges. 
A standard boundary element discretization (see e.g. [2,7]) of the back surface is performed using nine-noded quadrilateral elements and isoparametrical interpolation, thus enforcing the Hölder-continuity assumed in the derivation of the back surface BIE. With $N_{\text {node }}$ denoting the number of nodes on the truncated back surface $S_{T}$ after it has been discretized into $N_{e}$ elements, the number of unknowns on the back surface is $3 N_{\text {node }}$. The discretization of the COD given by Eq. (7) results in $3 N_{1} N_{2}$ unknown expansion coefficients $\alpha_{i n_{1} n_{2}}$, such that the total number of unknowns is $N_{d o f} \equiv 3\left(N_{\text {node }}+N_{1} N_{2}\right)$. The same number of equations is obtained by collocating the back surface BIE at each node point $\boldsymbol{y}_{n_{c}}$ on $S_{T}$ and by using the Galerkin method for the crack BIE, i.e. projecting the BIE on the $N_{1} N_{2}$ expansion functions. To solve the wave scattering problem it remains to evaluate all boundary element and crack integrals numerically, assemble the system of linear algebraic equations and solve for the unknown back surface node displacements and COD expansion coefficients.

It should be noted that after discretizing the crack integral equation using the Chebyshev functions the limit in front of the hypersingular integral in Eq. (3) can be evaluated analytically, since the expansion and projection leads to convergent integrals. The resulting integrals are also straightforward to compute numerically in an efficient way. The series expansion of the COD thus results in a solution method with an effective treatment of the hypersingularity.

The discretization of the back surface yields a very large coefficient matrix. This matrix may in general, as usual in the BEM, be both unsymmetric and fully populated. However, due to the spatial decay of the Green's tensors many off-diagonal matrix elements may be very small, and by setting all elements beneath a given threshold level to zero the system matrix becomes more or less sparse. A sparse solver can then be used, and the total computation times significantly reduced. For the examples considered in this paper a threshold resulting in a sparse system matrix with about $10 \%$ non-zero elements was used, and the equations were solved using the sparse parallel direct solver PARDISO [8].

As stated in the problem formulation, the shape of the back surface may be quite arbitrary. For the numerical results presented in this section two simple examples of back surfaces are used as illustrations, the first being a smooth transition in the form of a quarterwavelength sine function. The transition is from $x_{3}^{\mathrm{b}}=0$ to $x_{3}^{\mathrm{b}}=1.5 \mathrm{~mm}$ over the interval from $x_{1}^{\mathrm{b}}=-3 \mathrm{~mm}$ to $x_{1}^{\mathrm{b}}=3 \mathrm{~mm}$, independent of $x_{2}^{\mathrm{b}}$. The second example is a planar back surface with a local, smooth bump in the form of a product of two half-wavelength sine functions. The height of the bump is $1.5 \mathrm{~mm}$ and it is located at $\left(x_{1}^{\mathrm{b}}, x_{2}^{\mathrm{b}}\right) \in[-3,3] \times$ $[-2,2] \mathrm{mm}$ so that outside this domain the back surface is planar with $x_{3}^{\mathrm{b}}=0$. The peak of the bump is thus located at $\left(x_{1}^{\mathrm{b}}, x_{2}^{\mathrm{b}}\right)=(0,0)$. This second example is chosen to give an illustration of the influence on the signal response of a back surface with a very locally non-planar geometry.

In both cases the results of a pulse-echo testing sitation are given, i.e. the same probe is working as both transmitter and receiver. The probe is scanning along a scan surface parallel to the $x_{1}^{\mathrm{b}} x_{2}^{\mathrm{b}}$-plane and located at a distance of $10 \mathrm{~mm}$ from the lowermost part of the back surface. The probe is a square $10 \times 10 \mathrm{~mm} \mathrm{SV-probe} \mathrm{with} \mathrm{angle} \gamma_{\mathrm{t}}=-45^{\circ}$ and fluid coupling so that $\delta=0$. The results are for a single frequency of $1 \mathrm{MHz}$, i.e. most relevant for a narrow bandwidth probe.

For the steel material considered the Lamé constants are $\lambda=113.2 \mathrm{GPa}, \mu=$ $80.9 \mathrm{GPa}$ and the density is $\rho=7900 \mathrm{~kg} / \mathrm{m}^{3}$. To account for the effects of material damping the Lamé constants are given imaginary parts of $1 \%$ of the real parts. The crack is rectangular with sides $a_{1}=2 \mathrm{~mm}$ and $a_{2}=3 \mathrm{~mm}$ and the crack centre located at $\boldsymbol{d}^{\mathrm{b}}=\{-4,0,3\} \mathrm{mm}$. The Euler angles are $\varphi^{\mathrm{c}}=\psi^{\mathrm{c}}=0^{\circ}$ but $\theta^{\mathrm{c}}=60^{\circ}$.

Figure 2 shows the pulse-echo signal response as a function of probe position (i.e. the C-scan), for the case of the back surface with a transition from $x_{3}^{\mathrm{b}}=0$ to $x_{3}^{\mathrm{b}}=1.5 \mathrm{~mm}$. 


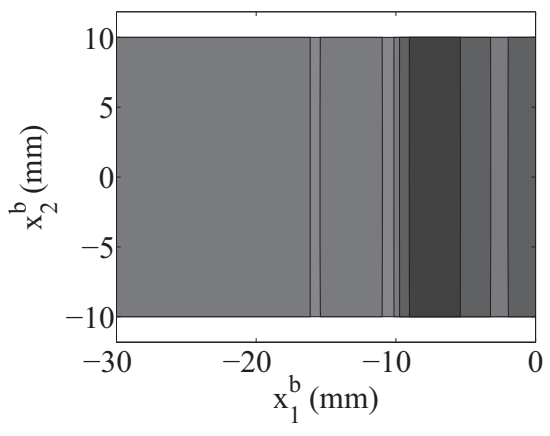

(a) Back surface signal response.

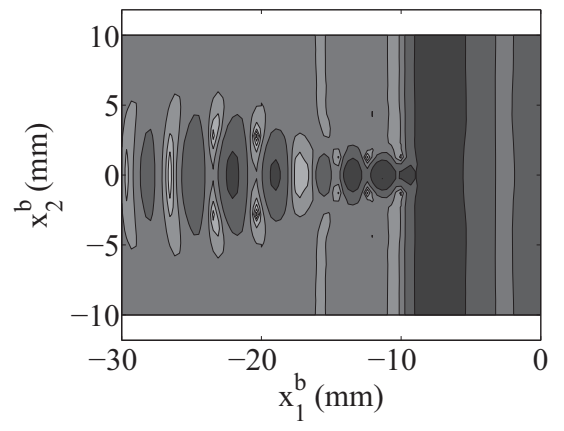

(b) Total signal response.

FIGURE 2. The echo amplitude as a function of probe position, for the first back surface geometry.

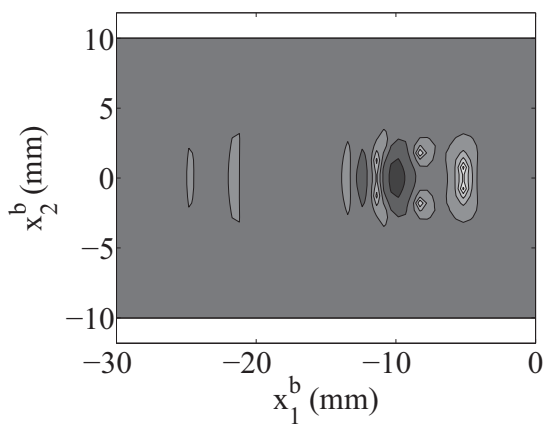

(a) Back surface signal response.

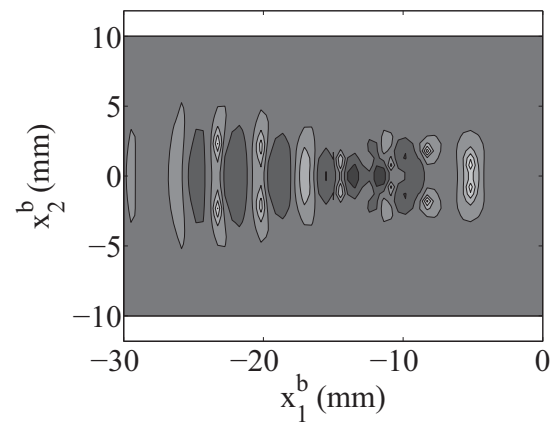

(b) Total signal response.

FIGURE 3. The echo amplitude as a function of probe position, for the second back surface geometry.

Figure 2(a) shows the back surface signal response, i.e. the response in the absence of the crack, whereas Fig. 2(b) shows the total signal response from both the back surface and the crack. Figure 3 shows the corresponding results for the second back surface type, with a local bump. In all the figures the same normalization is used, and a decibel (dB) scale is used with a $40 \mathrm{~dB}$ difference between black (strongest) and white (weakest), in steps of $5 \mathrm{~dB}$. As expected, the planar parts of the back surface give only a very weak signal response for the angled probe considered, and this response is seen to be equal for the planar parts of the back surface in all the figures. The independence of $x_{2}^{\mathrm{b}}$ for the first back surface type is also clearly seen in the signal response in Fig. 2(a). For the considered crack orientation the geometry is also symmetric about the line $x_{2}^{\mathrm{b}}=0$, and this symmetry is apparent also in the $\mathrm{C}$-scans. Finally, it can also be noted that the interaction of the back surface and the crack gives rise to quite complicated signal responses in Figs. 2(b) and 3(b).

\section{CONCLUSIONS}

In this paper a model of ultrasonic nondestructive testing is developed. The model includes transmitting and receiving ultrasonic contact probes located on a thick-walled component with a non-planar back surface and containing an interior rectangular crack. The action of the transmitting probe is accounted for in a probe model based on prescribing the traction on the scanning surface, while an electromechanical reciprocity argument is used to model 
the receiving probe and yield expressions for the signal response due to the crack and back surface. The wave scattering problem is solved by reformulating it as two coupled boundary integral equations for the unknown crack opening and back surface displacements. By using a combination of a series expansion of the crack opening displacement and a boundary element discretization of the back surface to solve the coupled integral equations, the hypersingularity in the crack BIE can be treated effectively while still allowing for a back surface of a general, complex geometry.

A few numerical results are presented, but these are for a single frequency computation only. A straightforward extension of the model is to include an inverse temporal Fourier transform to obtain the time traces (A-scans). Further, in real testing situations calibration against a standard scatterer such as a side-drilled hole is usually performed, and work is in progress to include such calibration in the model. Finally it is believed that defects which may be treated by the T-matrix method are well suited for incorporation in the present framework, and this is currently under investigation.

\section{ACKNOWLEDGEMENT}

The present work is sponsored by the Swedish Radiation Safety Authority (SSM) and this is gratefully acknowledged.

\section{REFERENCES}

1. P.J. Schafbuch, F.J. Rizzo, and R.B. Thompson, J. Nondestr. Eval. 9, 113-127 (1990).

2. M. Bonnet, Boundary Integral Equation Methods for Solids and Fluids, John Wiley \& Sons Ltd, Chichester, 1995.

3. P. Bövik and A. Boström, J. Acoust. Soc. Am. 102, 2723-2733 (1997).

4. J. Westlund and A. Boström, Wave Motion 33, 1103-1112 (2010).

5. A. Boström and H. Wirdelius, J. Acoust. Soc. Am. 97, 2836-2848 (1995).

6. B.A. Auld, Wave Motion 1, 3-10 (1979).

7. J. Domínguez, Boundary Elements in Dynamics, Computational Mechanics Publications, Southampton, 1993.

8. O. Schenk and K. Gärtner, J. Fut. Gen. Comp. Sys. 20, 475-487 (2004). 